Proceedings

\title{
Dynamic Mechanical Simulation of Miniature Silicon Membrane during Air Blast for Pressure Measurement ${ }^{+}$
}

Julien Veyrunes 1, Jérôme Riondet 2,3, André Ferrand 1, Maylis Lavayssière ${ }^{3}$, Alexandre Lefrançois ${ }^{3}$, Jérôme Luc ${ }^{3}$, Hervé Aubert ${ }^{2}$ and Patrick Pons ${ }^{2, *}$

1 ICA, Université de Toulouse, 31400 Toulouse, France; veyrunes@etud.insa-toulouse.fr (J.V.); andre.ferrand@insa-toulouse.fr (A.F.)

2 LAAS-CNRS, Université de Toulouse, CNRS, INP, 31400 Toulouse, France; jriondet@laas.fr (J.R.); aubert@laas.fr (H.A.)

3 CEA-DAM, 46500 Gramat, France; maylis.lavayssiere@cea.fr (M.L.); alexandre.lefrancois@cea.fr (A.L.); jerome.luc@cea.fr (J.L.)

* Correspondence: ppons@laas.fr; Tel.: +33-5-61-33-64-63

+ Presented at the Eurosensors 2018 Conference, Graz, Austria, 9-12 September 2018.

Published: 18 December 2018

Abstract: The development of new ultra-fast sensors for pressure air blast monitoring requires taking into account the very short rise time of pressure occurring during explosion. Simulations show here that the dynamic mechanical behavior of membrane-based sensors depends significantly on this rise time when the fundamental mechanical resonant frequency of the membrane is higher than $10 \mathrm{MHz}$.

Keywords: air blast; pressure sensor; dynamic behavior; silicon membrane

\section{Introduction}

The real time and dynamic measurements of pressure during air blasts is very challenging due to the abrupt variation of pressure from the atmospheric pressure to the so-called overpressure peak $P_{\max }$ (between few bars and several ten of bars) with very short rise time $t_{m}(<100 \mathrm{~ns})$ (Figure 1). For accurate measurement of $P_{\max }$, sensors with high fundamental mechanical resonant frequency $F_{o}$ are then required [1].
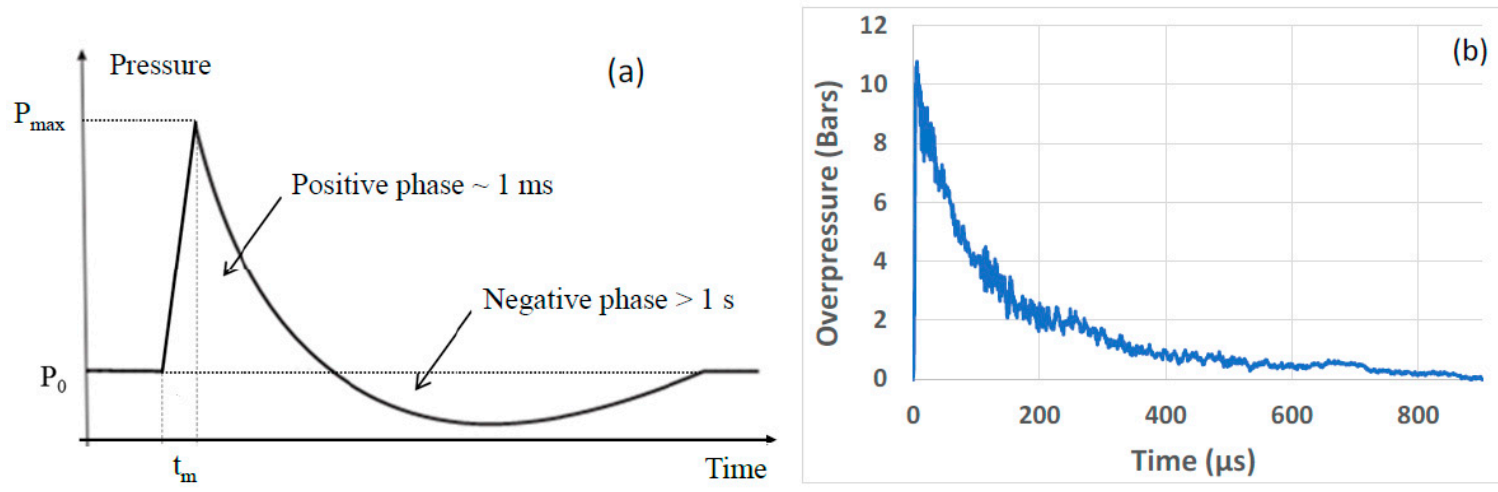

Figure 1. Typical dynamic pressure variation during an air blast experiment: (a) Illustration of the different phases and (b) example of pressure measurement at $1 \mathrm{~m}$ from $1 \mathrm{kgTNT}$ by using a Tourmaline piezoelectric sensor. 
When simulating a pressure sensor for which $F_{0} \ll 1 / \mathrm{tm}$, the rise-time is assumed to be close to zero. However, we show here that this assumption is no more valid when performing the mechanical simulation of a sensor for which $\mathrm{F}_{\mathrm{o}}>10 \mathrm{MHz}$.

\section{Sensor Description and Simulation Conditions}

As shown in Figure 2, the proposed sensor is based on a miniature rectangular silicon membrane $(5 \mu \mathrm{m} \times 30 \mu \mathrm{m} \times 90 \mu \mathrm{m})$ with four piezoresistive gauges located at its center [1].

Simulations were performed using Abaqus software [2] for real membrane clamping conditions. Considering the gauges dimensions, the stresses in the gauge areas are close to ones calculated at the membrane center (error lower than $7 \%$ on the sensor response). Dynamic behavior of the sensor is then modelled by the differential stress $\Delta \sigma$ given by Equation (1) at the center of the membrane, and normalized by the static pressure:

$$
\Delta \sigma=\sigma_{l}-\sigma_{t}
$$

where $\sigma_{l}$ (resp., $\sigma_{t}$ ) is the stress applied to the gauge parallel (resp., to the gauge perpendicular) to the current in the gauge.

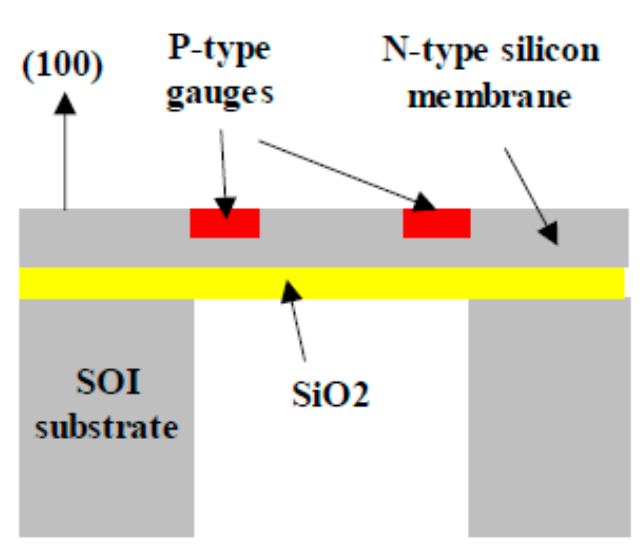

(a)

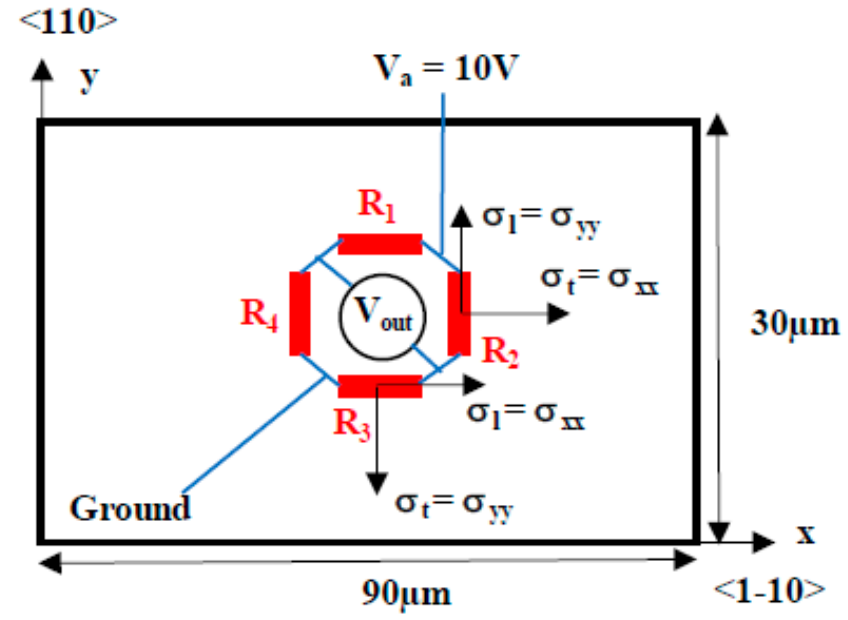

(b)

Figure 2. (a) Cross sectional view of the sensor; (b) Wheatstone bridge on the rectangular membrane.

The fundamental mechanical resonant frequency $\mathrm{F}_{\mathrm{o}}$, obtained from harmonic module of Abaqus software, is of $32.7 \mathrm{MHz}$ and consequently $1 / \mathrm{F}_{\mathrm{o}}$ is close to $30 \mathrm{~ns}$.

Due to the short reaction time tr of the sensor $(<\mathrm{few} \mu \mathrm{s}$, see Figure 5$)$, we assume here that the pressure profile (shown on Figure 1) can be modelled by the Heaviside step function (the decrease of pressure is lower than $2 \%$ after $1 \mu \mathrm{s})$.

Moreover, assuming that the acoustic damping is predominant [3], the quality factor $Q$ of the membrane is inversely related to the equivalent pressure $P_{e}$ applied on both sides of the membrane as shown in Equation (2).

$$
Q \cong \frac{95}{P_{e}(\text { bar })}
$$

Abaqus simulations indicates that the pressure $P_{e}$ is the average pressure applied between the two membrane sides. Table 1 reports the $Q$ factor for typical applied pressure on one side of the membrane while the other side is in vacuum. We observe that the $Q$ factor decreases rapidly and is lower than 20 for the applied pressure greater than 10 bars. 
Table 1. $Q$ factor versus absolute pressure from Equation (2).

\begin{tabular}{ccccccc}
\hline Pressure (Bar) & $\mathbf{2}$ & $\mathbf{5}$ & $\mathbf{1 0}$ & $\mathbf{2 0}$ & $\mathbf{3 0}$ & $\mathbf{4 0}$ \\
\hline $\boldsymbol{Q}$ & 95 & 38 & 19 & 9.5 & 6.3 & 4.8 \\
\hline
\end{tabular}

For a shock wavefront normally incident upon the membrane surface, the dynamic mechanical response to a linear variation of pressure was obtained from the Second Order Transfer Function (SOTF) where the rise time differs from zero (Equation (3)). This model is consistent with Abaqus simulations results, as it can be noticed from Figure 3. We can observe that the difference is lower than $4 \%$ after the rise time. Consequently, the effects on sensor reaction time can be neglected.

$$
\begin{aligned}
\mathrm{R}_{n}(t)=\frac{1}{t_{m}}\{ & t-2 \cdot B+e^{-A \cdot t}\left[2 \cdot B \cdot \cos (\Omega \cdot t)+\frac{2 \cdot \xi^{2}-1}{\Omega} \sin (\Omega \cdot t)\right] \\
& -U\left(t-t_{m}\right)\left[t-t_{m}-2 \cdot B \cdot e^{-A\left(t-t_{m}\right)} \cdot\left(2 \cdot B \cdot \cos \left(\Omega\left(t-t_{m}\right)\right)\right.\right. \\
& \left.\left.+\frac{2 \cdot \xi^{2}-1}{\Omega} \sin \left(\Omega\left(t-t_{m}\right)\right)\right]\right\}
\end{aligned}
$$

where $\xi$ is the damping factor, $\mathrm{A}=\xi \cdot \omega_{o}, \mathrm{~B}=\frac{\xi}{\omega_{o}}, \Omega=\omega_{o} \sqrt{1-\xi^{2}}, \omega_{o}=1 / F_{0}$. and $U(t)=0$, if $t<0, U(t)=\frac{t}{t_{m}}$, if $0<t<t_{m}, U(t)=1$ if $t>t_{m}$.
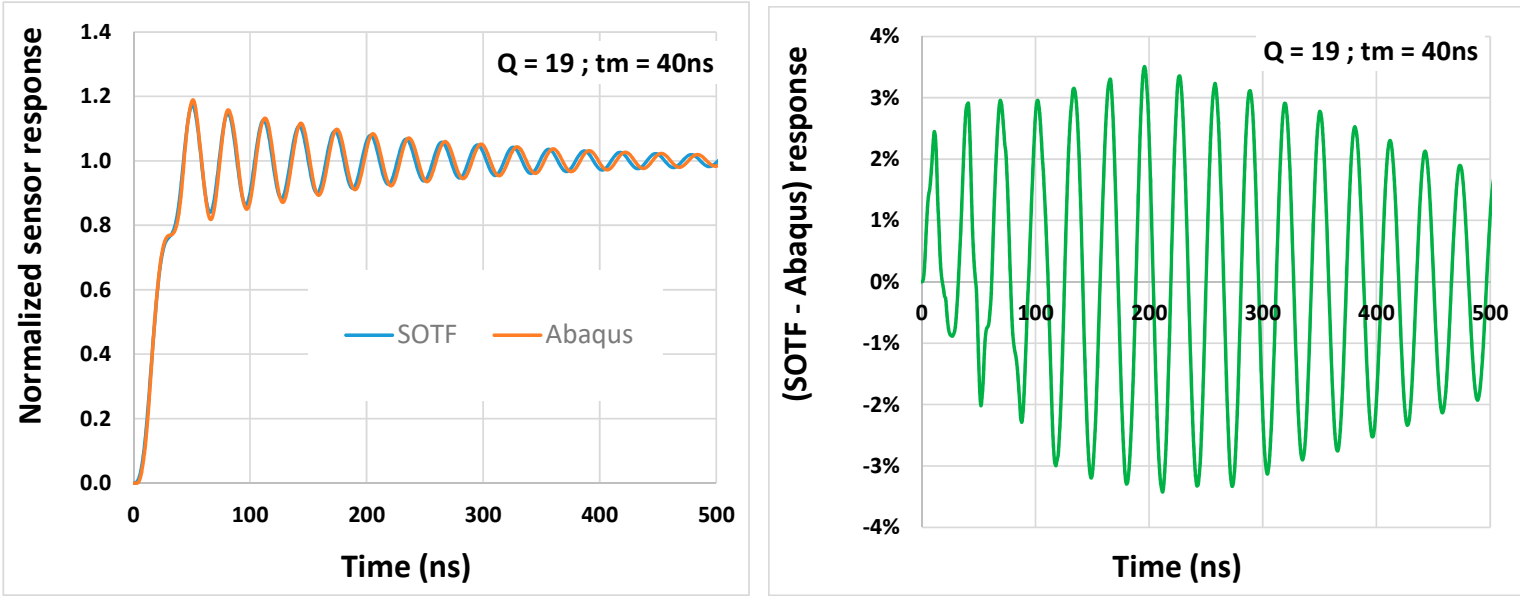

Figure 3. Comparison of SOTF model and Abaqus simulation results for the dynamic response.

\section{Results}

Figure 4 displays an example of the dynamic mechanical response of the membrane for rise time $\mathrm{t}_{\mathrm{m}}$ up to $100 \mathrm{~ns}$ and for a $Q$ factor of 19 . The response is normalized by the static value. The reaction time $t_{r}$ of the sensor (00B $15 \%$ of the static response) is extracted from these figures for $Q$ factor between 5 and 100 (Figure 5). The following observations can be made:

i. for $t_{m}<1 / \mathrm{F}_{\mathrm{o}}$, we retrieve the classical damped oscillation for which $t_{r}$ is mainly driven by the $Q$ factor $\left(t_{r} \cong Q / F_{0}\right)$;

ii. as $t_{m}$ increases, the reaction time tr decreases and, due to the decreasing of the fundamental mode amplitude (Figure 6), we obtain $t_{r} \cong t_{m}$ when $t_{m}=1 / \mathrm{F}_{0}$;

iii. when $t_{m}=n / \mathrm{F}_{\text {o where }} n$ is a natural number, no oscillation occurs and $t_{r}$ is close to $t_{m}$;

iv. when $t_{m}>1 / \mathrm{F}_{\mathrm{o}}$, small oscillations appear during the rise time.

The reduction of the reaction time related to the increase of the rise time is given in Figure 7 (reaction time for $t_{m}=0$ is taken as reference). We can observe that for pressure rise time tm greater than $20 \mathrm{~ns}$, the sensor reaction time may be underestimated almost by $20 \%$ compared with the zero pressure rise- time $\left(t_{m}=0\right)$ assumption. This error may reach $100 \%$ when $t m$ is close to $n / F_{0}$. 

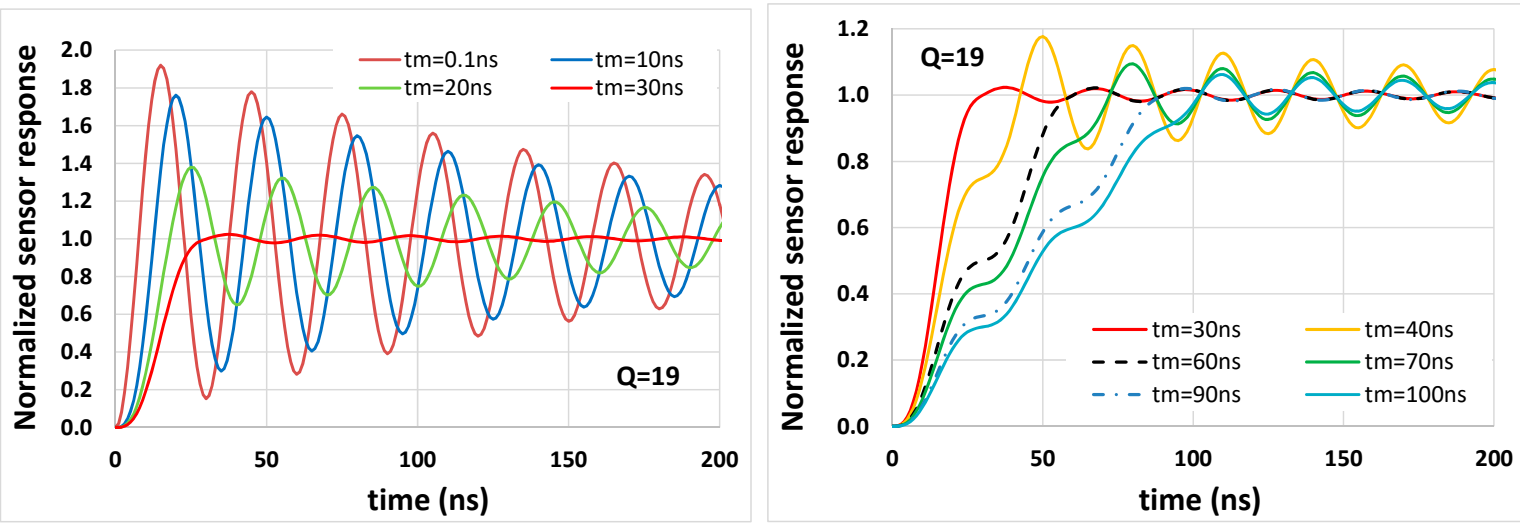

Figure 4. Dynamic sensor response for different rise times.
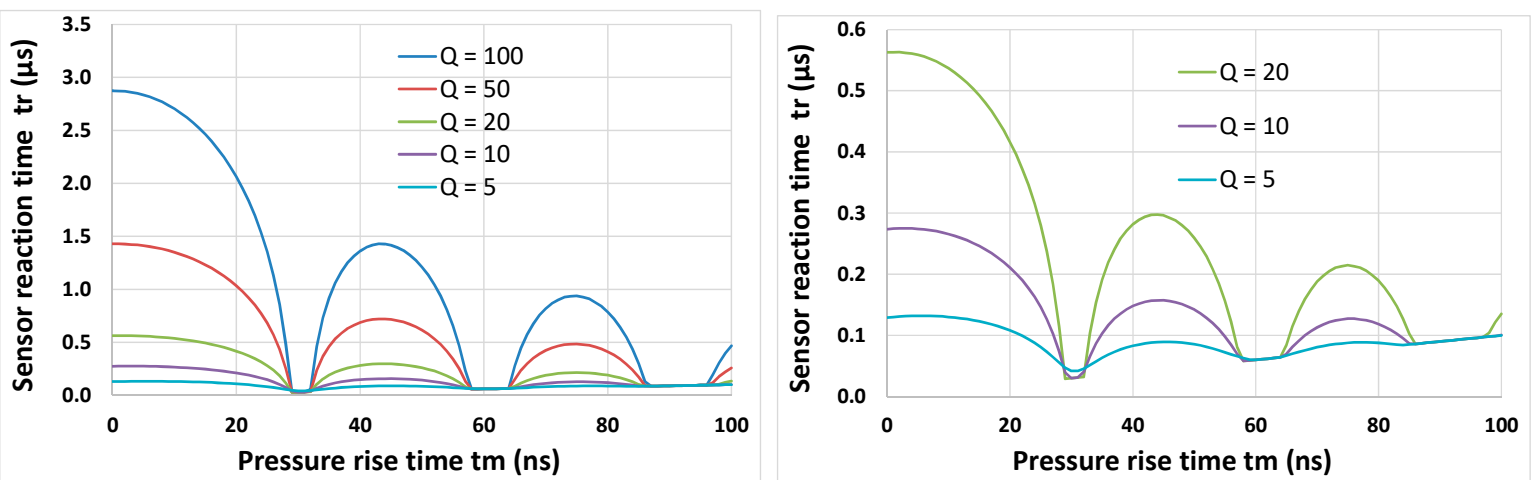

Figure 5. Sensor reaction time versus pressure rise time for different $Q$ factor.

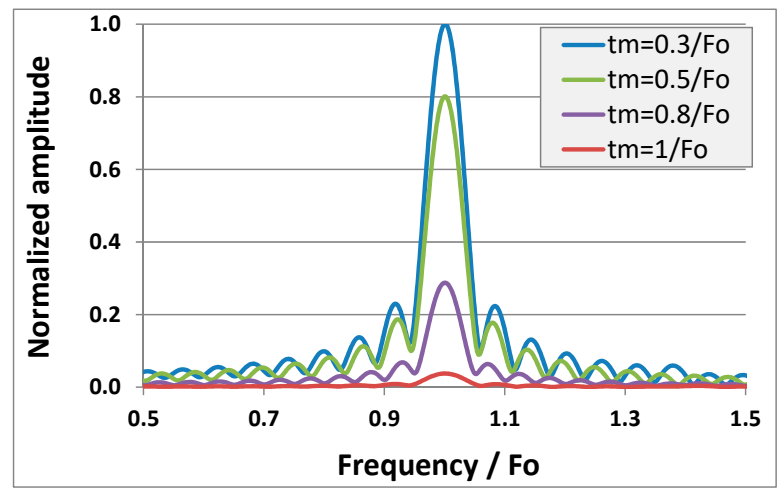

Figure 6. Amplitude of fundamental mode versus $t_{m}$ (Abaqus simulations results).

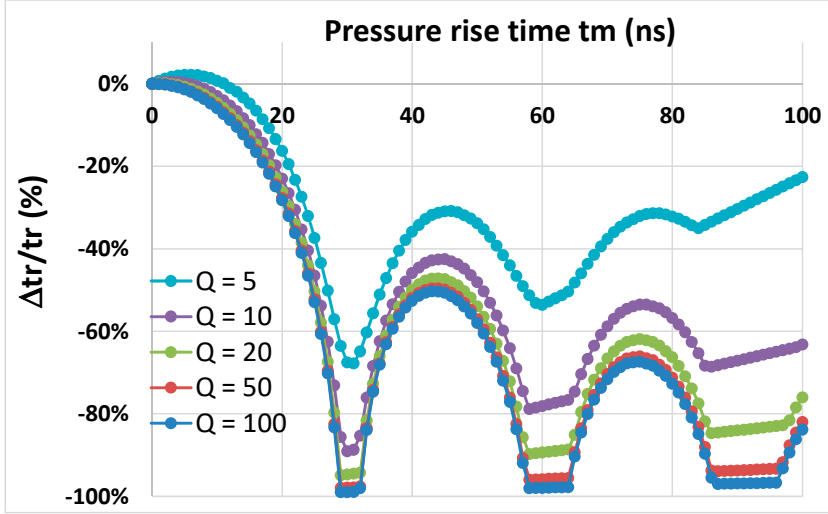

Figure 7. Relative sensor reaction time versus pressure rise time for different $Q$ factors. 


\section{Conclusions}

The dynamic mechanical behavior of membrane-based piezoresitive sensors was modelled by a second order transfer function with non-zero pressure rise time. For miniaturized silicon membrane $(5 \mu \mathrm{m} \times 30 \mu \mathrm{m} \times 90 \mu \mathrm{m})$ the fundamental resonant frequency $\mathrm{F}_{\mathrm{o}}$ is of $33 \mathrm{MHz}$. The results show that pressure rise time tm larger than $1 /\left(3 \mathrm{~F}_{\mathrm{o}}\right)$ plays a crucial role for the accurate estimation of the sensor reaction time. Consequently, when designing ultra-fast sensors for pressure air blast monitoring, the increase of frequency $F_{0}$ for a given rise time is expected to provide a significant reduction of the sensor reaction time.

Acknowledgments: This work was partially funded by Occitanie Region (France) through the COCNANO project.

Conflicts of Interest: The authors declare no conflict of interest. The founding sponsors had no role in the design of the study; in the collection, analyses, or interpretation of data; in the writing of the manuscript, and in the decision to publish the results.

\section{References}

1. Riondet, J.; Coustou, A.; Aubert, H.; Pons, P.; Lavayssière, M.; Luc, J.; Lefrançois, A. Design of air blast pressure sensors based on miniature silicon membrane and piezoresistive gauges. J. Phys. Conf. Ser. 2017, 922, 012019.

2. Overview of ABAQUS-Explicit. Available online: http://imechanica.org/files/0-overview\%20Explicit.pdf (accessed on 3 December 2018).

3. Prak, A.; Blom, F.R.; Elwenspoek, M.; Lammerink, T.S.J. Q-factor and frequency shift of resonating silicon diaphragms in air. Sens. Actuators A 1991, 27, 691-698.

(C) 2018 by the authors. Licensee MDPI, Basel, Switzerland. This article is an open access article distributed under the terms and conditions of the Creative Commons Attribution (CC BY) license (http://creativecommons.org/licenses/by/4.0/). 